\title{
Production and Characterization of Carbon Molecular Sieves from Bituminous Lafia-Obi Nasarawa Coal by Pore Size Modification with Spent Engine Oil
}

\author{
E. Bala* ${ }^{\S}$, O.R. Momoh, B.O. Aderemi and B.J. El-Yakubu \\ Department of Chemical Engineering, Ahmadu Bello University Zaria, Nigeria.
}

Received 13 February, revised 16 October 2018, accepted 29 November 2018.

\begin{abstract}
In this work, a carbon molecular sieve (CMS) was produced from a bituminous Lafia-Obi Nasarawa coal. An initial activated carbon (AC) sample was prepared via chemical activation from the coal, from which the CMS samples were then produced through heat treatment processes and carbon deposition from spent engine oil. Spent engine oil was pyrolyzed in order to deposit carbon at the pore mouth of coal-based AC to yield CMS. The effect of reaction temperature and holding time variation on the surface area, micropore pore volume and pore size of CMS was studied. Reaction temperature was varied at 400,550 and $700{ }^{\circ} \mathrm{C}$ while holding time was varied at 30 and $60 \mathrm{~min}$. The Brunauer-Emmett-Teller (BET) method was used to calculate the surface areas, while the Dubinin-Radushkevich (DR) and Horváth-Kawazoe (HK) methods were used to determine the micropore volumes and pore sizes of the AC and CMS, respectively. The CMS sample with the largest BET surface area $\left(5.824 \mathrm{~m}^{2} \mathrm{~g}^{-1}\right), \mathrm{DR}^{-}$ micropore volume $\left(0.172 \mathrm{~cm}^{3} \mathrm{~g}^{-1}\right)$ and HK pore size $\left(6.317 \AA\right.$ ) were obtained at $700{ }^{\circ} \mathrm{C}$ reaction temperature and 60 min holding time. In addition to this, a molecular sieving ability test to separate benzene from a mixture of benzene and o-xylene in solution was carried out on the AC and CMS, with the CMS having a selective benzene percentage uptake of $61.36 \%$.
\end{abstract}

KEYWORDS

Activated carbon (AC), carbon molecular sieves (CMS), carbon deposition, reaction temperature, holding time.

\section{Introduction}

The separation of molecules from mixtures into their various components constitutes a significant cost in industry from an energy and environmental perspective. ${ }^{1}$ Some very common and important molecular separations in the chemical industry today involve the separation of air, petrochemicals and alcohols by processes requiring large amounts of energy with its attendant environmental pollution implications. Owing to this, there is an increasing interest in the use of adsorbents operating at moderate process conditions to achieve these molecular separations with reduced energy consumption, and minimal negative impact on the environment. CMS are a special type of AC able to discriminate molecules on the basis of their size and shape. ${ }^{2}$ They are microporous materials, capable of carrying out molecular separations based on the differences in the rates of adsorption of the adsorbate molecules. ${ }^{3}$ The choice of the raw material constitutes the first important step for CMS production. Generally, certain bituminous coal qualities and coked coconut shells have proven to be suitable raw materials for industrial production. ${ }^{4} \mathrm{CMS}$ can be synthesized from various carbon-containing precursors such as lignocellulosic materials, coals, carbon fibres and pitch by different methods like: (i) pyrolysis (ii) controlled gasification of chars to increase the pore size, (iii) thermal treatment of carbon precursor to modify the pore size, (iv) chemical vapour deposition (CVD) of carbon in the mouth of the pores and, (v) modification of coals by mixing with tars and resins and subsequent carbonization. ${ }^{5}$ Another approach for narrowing the pore size is by the pyrolysis of an organic precursor previously impregnated in the AC. AlcañizMonge et al. ${ }^{4}$ analyzed this approach and prepared CMS from the co-carbonization of a bituminous coal impregnated with a * To whom correspondence should be addressed. E-mail: balazibya@yahoo.com slurry of coal tar pitch. Bituminous coal, due to its microstructure and surface composition, has been proposed as a precursor for CMS by many researchers. ${ }^{4,6-7}$ To optimize the preparation process, the modification of the porosity (pore blocking) of the starting $\mathrm{AC}$, the molecular sieving properties of the CMS obtained by this method are analyzed. A report by the World Energy Council ${ }^{8}$ puts Nigeria's total proven coal reserves at 344 million tons, most of which fall within the rank of bituminous or sub-bituminous. Of the total Nigerian coal deposits, the Lafia-Obi basin has an estimated 21 million tons of bituminous coal reserves. ${ }^{9}$ This makes it an attractive economically viable precursor for the production of CMS. This study investigates the effect of reaction temperature and holding time variation on the surface parameters of CMS made from bituminous Lafia-Obi Nasarawa coal using spent engine oil as a carbon deposition agent.

\section{Experimental}

The schematic diagram of the experimental setup is shown in Fig. 1. The main component of the system is a stainless steel reactor $(23.5 \mathrm{~cm}$ internal diameter). The reactor is designed for a batch operation and it is heated within an enclosing electronic tube furnace.

Prior to the carbonization process, a proximate and elemental analysis was carried out on the coal raw material to determine its ranking and the results obtained are presented in Tables 1 and 2 . All samples were characterized in terms of surface area, micropore volume and pore size by $\mathrm{N}_{2}$ adsorption at $77 \mathrm{~K}$ in a Quantachrome Autosorb Automated Gas Sorption System. The sample was placed inside a tube and a glass bulb was inserted inside the tube. The bulb was inserted by slanting the sample tube almost to a horizontal position. Before an experiment began, the 


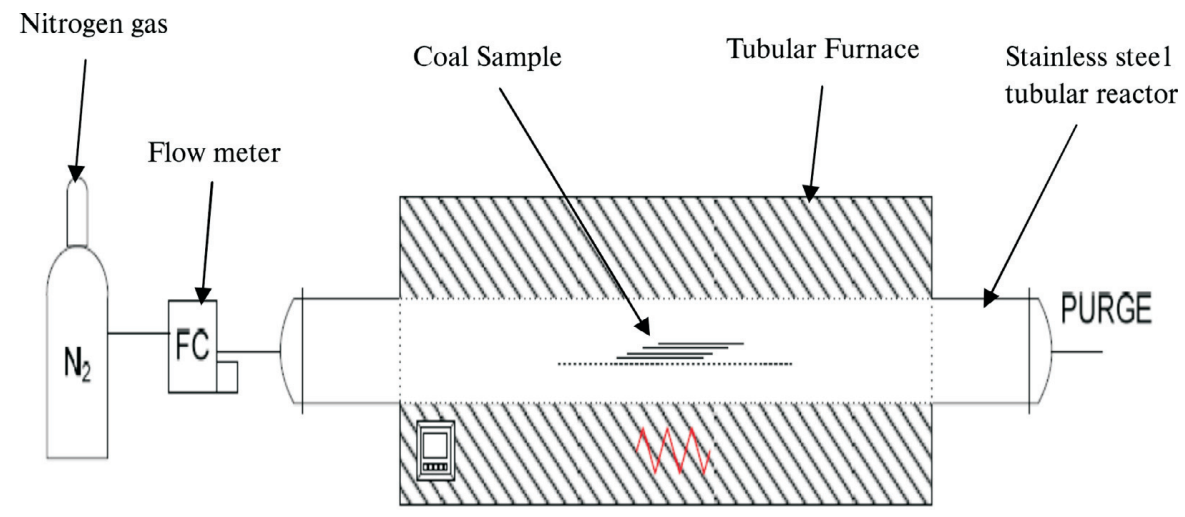

Figure 1 Experimental set-up for the production of the AC and CMS samples.

adsorbents were degassed (10-4 mm Hg) at $393 \mathrm{~K}$. The surface area of the samples was measured based on the BET method. The DR method was applied to calculate the micropore volume, and the pore size was investigated based on the HK method.

\subsection{Preparation of Activated Carbon (AC)}

A size reduction of the raw coal material was carried out to a $2 \mathrm{~mm}$ aperture. A $100 \mathrm{~g}$ coal sample was then weighed and mixed with $400 \mathrm{~g}$ of phosphoric acid $\left(\mathrm{H}_{3} \mathrm{PO}_{4} 85 \% \mathrm{v} / \mathrm{v}\right)$, continuously stirred on a constant temperature magnetic stirrer at $80^{\circ} \mathrm{C}$ for a period of $3 \mathrm{~h}$ to ensure maximum contact and mixing between the acid and sample. The resulting slurry was then placed in an oven to dry at $105^{\circ} \mathrm{C}$ for a period of $24 \mathrm{~h} .{ }^{10} 50 \mathrm{~g}$ of the phosphoric acid impregnated coal was packed into a tubular stainless steel vessel (ID $24 \mathrm{~mm}, \mathrm{~L} 30 \mathrm{~cm}$ ) and placed in an electronic furnace. The furnace was heated under nitrogen atmosphere at a flowrate of $5 \mathrm{~L} \mathrm{~min}^{-1}$ and a heating rate of $6{ }^{\circ} \mathrm{C} \mathrm{min}^{-1}$ up to a final temperature of $600^{\circ} \mathrm{C}$, and then held at this temperature for $2 \mathrm{~h}$ after which the furnace was switched off and allowed to cool under nitrogen till room temperature. The cooled coal sample was then thoroughly washed in distilled water while constantly monitoring the $\mathrm{pH}$ level of the solution until a neutral $\mathrm{pH}$ was observed, before being oven-dried at $105^{\circ} \mathrm{C}$ for $12 \mathrm{~h}$ to eliminate all moisture. ${ }^{11} \mathrm{~A}$ BET analysis and molecular sieving ability test to determine the ability of the AC to selectively adsorb benzene over o-xylene from a binary solution of the two was then carried out on the resulting AC sample.

\subsection{Preparation of the Carbon Molecular Sieves (CMS)}

$10 \mathrm{~g}$ of $\mathrm{AC}$ was weighed and transferred into a beaker containing $5 \mathrm{~g}$ of spent engine oil. The mixture was then rigorously stirred for a period of $30 \mathrm{~min}$ to attain homogeneity, placed in a covered vessel and left to stand at room temperature for a period of $24 \mathrm{~h} .5 \mathrm{~g}$ of the engine oil impregnated AC sample was weighed, placed in the tubular stainless steel vessel and heated in the electric furnace under nitrogen atmosphere at a flowrate of $5 \mathrm{~L} \mathrm{~min}{ }^{-1}$. With the AC sample being heated at a rate of $6{ }^{\circ} \mathrm{C} \min ^{-1}$, the temperature of the furnace was raised to $400{ }^{\circ} \mathrm{C}$ and kept at this temperature for 30 and $60 \mathrm{~min}$. This experiment was repeated at temperatures of 550 and $700{ }^{\circ} \mathrm{C}$ with holding times of 30 and $60 \mathrm{~min}$ for all reaction temperatures. The resulting CMS samples were then taken for a BET analysis and molecular sieving ability test to determine their ability to selectively adsorb benzene over o-xylene from a binary solution of the two.

\subsection{Molecular Sieve Ability Test for AC and CMS}

Benzene and o-xylene solutions of $99.5 \%(\mathrm{v} / \mathrm{v})$ purity were used to prepare the standard solutions used in the plotting of the absorbance versus concentration calibration curves to determine equilibrium concentrations. The initial stock solution concentrations of the reagents were determined to be $11.21 \mathrm{M}$ for benzene and $8.25 \mathrm{M}$ for o-xylene. Standard solutions of varying concentrations ranging from $0.1 \mathrm{M}$ to $0.5 \mathrm{M}$ were prepared for both reagents using methanol of $98.5 \%(\mathrm{v} / \mathrm{v})$ purity as a solvent. All solutions were then taken for UV spectrophotometry analysis. Methanol $98.5 \%(\mathrm{v} / \mathrm{v})$ was added to a solution mixture containing $0.39 \mathrm{~mL}$ of $11.25 \mathrm{M}$ benzene and $0.53 \mathrm{~mL}$ of $8.25 \mathrm{M}$ o-xylene to make up a total volume mixture of $10 \mathrm{~mL}$. The change in concentrations of benzene and o-xylene in solution were then determined from their respective calibration curves (see Fig. 5). This mixture was divided into two equal parts and poured into two $50 \mathrm{~mL}$ beakers, one containing $0.25 \mathrm{~g}$ of AC and the other $0.25 \mathrm{~g}$ of CMS 700/60 (The CMS sample produced at $700{ }^{\circ} \mathrm{C}$ and $60 \mathrm{~min}$ holding time). The beakers were then placed in an agitator for $24 \mathrm{~h}$ until equilibrium was attained after which $2 \mathrm{~mL}$ was drawn from each solution and taken for UV spectrophotometry analysis.

\section{Results and Discussion}

\subsection{BET Results for AC}

The AC sample had a surface area value of $596.2 \mathrm{~m}^{2} \mathrm{~g}^{-1}$, a DR micropore volume of $0.173 \mathrm{~cm}^{3} \mathrm{~g}^{-1}$ and HK pore size of $9.432 \AA$. The development of porosity in activated carbons made from bituminous coals has been discussed by numerous researchers. ${ }^{1,6,10}$

\subsection{Proximate and Elemental Analysis of Raw Coal Sample}

From Table 1 the proximate analysis of the raw coal sample indicates that it has a moisture content of $14.1 \%$, a fixed carbon of $63.61 \%$, an ash content of $14.07 \%$ and a volatile matter of $8.22 \%$. Also, by way of elemental analysis as shown in Table 2 , the coal sample has a carbon content of $76.2 \%$, a hydrogen content of $5.89 \%$, nitrogen of $2.19 \%$, with sulphur and oxygen at $5.9 \%$ and $9.82 \%$, respectively. According to Speight ${ }^{12}$ the most important parameters used in determining the ranking of coals are the fixed carbon content for the proximate analysis and the percentage carbon content for the elemental analysis. Since both

Table 1 Proximate analysis of raw coal sample.

\begin{tabular}{lc}
\hline Substance in sample & Percentage in sample/\% \\
\hline Moisture content & 14.1 \\
Fixed carbon & 63.61 \\
Ash & 14.07 \\
Volatile matter & 8.22 \\
\hline
\end{tabular}


Table 2 Elemental analysis of Nasarawa coal.

\begin{tabular}{lc}
\hline Element & Percentage in sample/\% \\
\hline Carbon & 76.20 \\
Hydrogen & 5.89 \\
Nitrogen & 2.19 \\
Sulphur & 5.90 \\
Oxygen & 9.82 \\
\hline
\end{tabular}

results of the fixed carbon and percentage carbon content are $63.61 \%$ and $76.2 \%$, respectively, the coal sample used for this study can be ranked as being bituminous, and hence can serve as a suitable precursor for the production of CMS.?

\subsection{Effect of Reaction Temperature and Holding Time \\ Variation on Surface Area of CMS}

Figure 2 shows the effect of reaction temperature and holding time on the development of the surface area of the various CMS samples. The data in the figure show a progressive increase in surface area values for all CMS samples produced with increasing reaction temperatures and times after an initial drop from that of the AC precursor (see Table 1). This could be as a result of excessive pore mouth blocking by the unpyrolyzed oil and char formed during the pyrolytic decomposition of the spent engine oil molecules embedded within the pore structure. ${ }^{13-14}$ At both holding times of 30 and $60 \mathrm{~min}$, an increase in surface area was observed as pyrolysis temperature was increased. It can be postulated that with higher pyrolysis temperatures, lighter hydrocarbons are formed to the detriment of residue (char), which in turn escape the pores and get deposited as carbon at the pore mouths and exterior of the CMS pore structure. This in turn leads to some mesopores being converted to micropores, thus enhancing surface area development. ${ }^{15}$

An increase in residence time equally led to a corresponding increase in surface areas at all temperatures, due to fact that a longer deposition time is necessary for the complete pyrolytic decomposition and deposition of the spent oil molecules. ${ }^{16-17}$

\subsection{Effect of Reaction Temperature and Time Variation on DR Pore Volume of CMS}

Reaction temperature and time variation was found to have a significant effect on the DR pore volume of the CMS. During the reaction, a rigorous control of reaction temperature and time is necessary so as to avoid the pore mouth blockage that occurs when excessive carbon is deposited within the pore structure of the CMS..$^{18}$ In light of this, reaction temperatures were kept at 400,550 and $700{ }^{\circ} \mathrm{C}$, while residence times were varied at 30 and $60 \mathrm{~min}$. This is illustrated in Fig. 3.

A reduction in the pore volume for all CMS samples from that of the AC sample was observed. It can be proposed that at

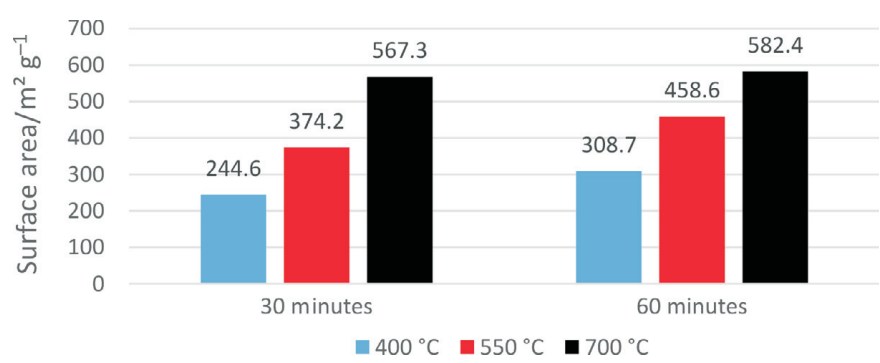

Figure 2 Effect of reaction temperature and holding time variation on surface area of CMS.

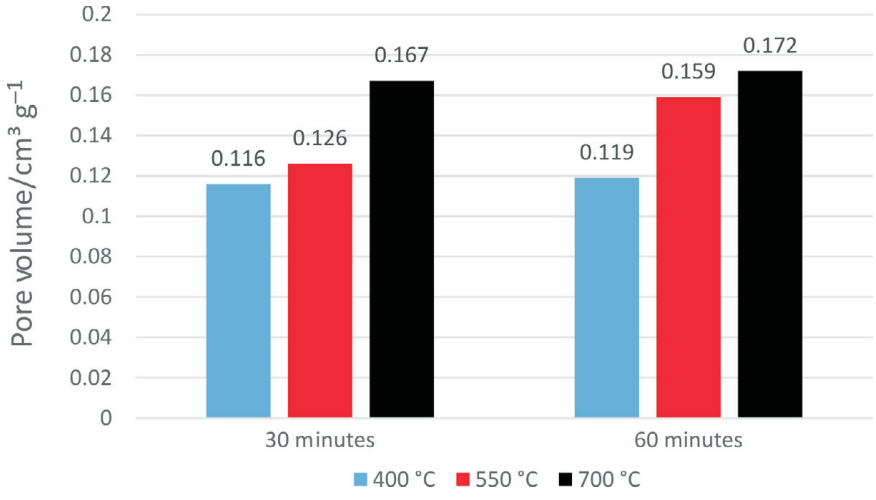

Figure 3 Effect of reaction temperature and holding time variation on DR pore volume of CMS.

relatively lower reaction temperatures, the molecules from the oil formed in the vapour phase Are 'pyrolytically' deposited in the interior of the pores and not at the pore mouths, leading to the excessive coke formation within the pores and a lower pore volume (adsorption capacity). ${ }^{15}$ As reaction temperature and time are increased, an improvement in pore volume can be observed. This could be because as the transition of the spent engine oil molecules from the liquid to gas phase takes place more rapidly, the pyrolytic cracking and deposition takes place more at the pore mouths and exterior of the pore structure, leading to a considerably less amount of coke formation within the pores. ${ }^{13-14}$ Also with longer holding times, the heat transfer within the oil molecules is more enhanced leading to a more rapid phase change and carbon deposition at pore mouths and the exterior. ${ }^{19}$

\subsection{Effect of Reaction Temperature and Holding Time Variation on HK Pore Size of CMS}

The pore size of a CMS is a very important surface characteristic, owing to the fact that it confers upon the CMS the all-important quality of selectivity. It is at this point that the effect that reaction temperature and holding time have on the deposition of carbon on the molecular sieve's surface and thus its pore size modification, come into play. ${ }^{15}$ From Fig. 4 it can be seen that at all reaction temperatures and times, the values of the pore sizes of all CMS samples steadily decline from that of the AC precursor from which they were made. As is observed, when reaction temperature and time get increased, deposition of carbon from the pyrolytic decomposition of hydrocarbon oil molecules occurs more at the pore mouths and exterior than inside the pore structure, leading to progressive pore size narrowing.

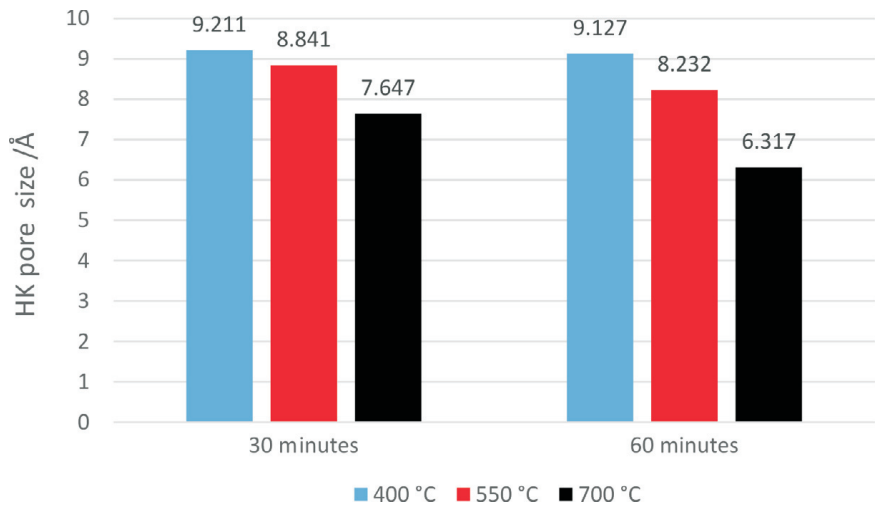

Figure 4 Effect of reaction temperature and holding time variation on HK pore size of CMS. 


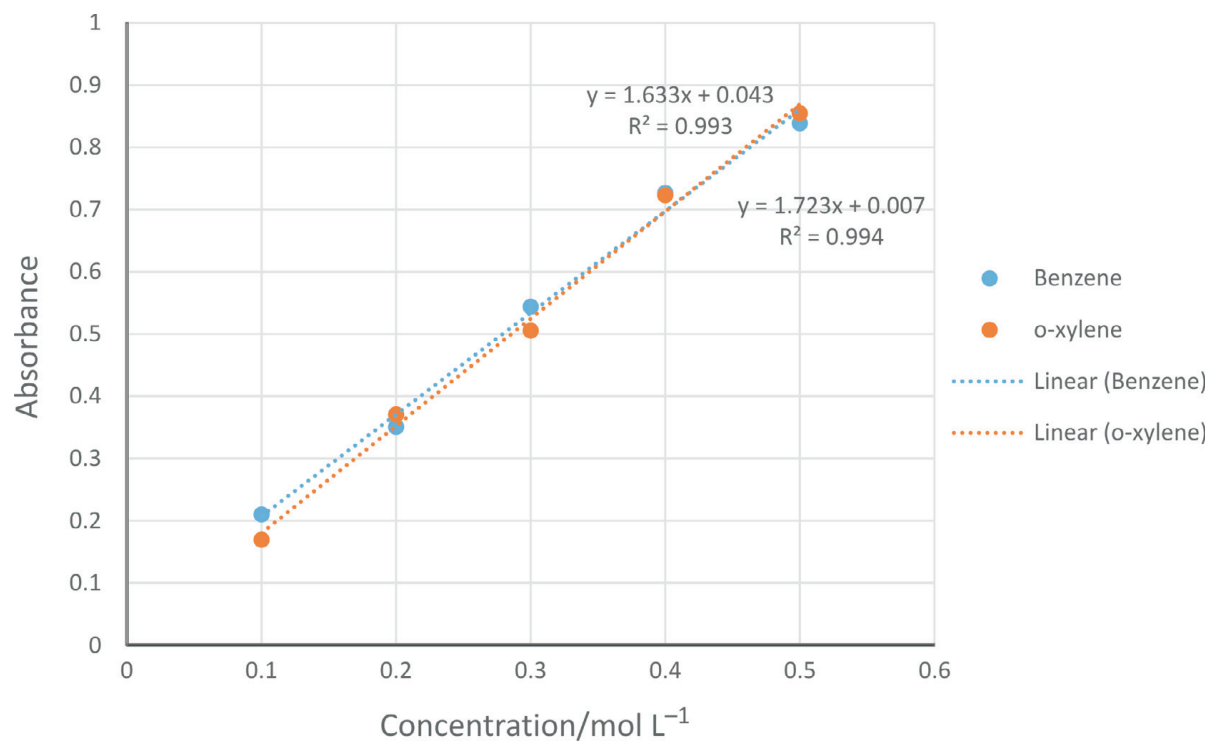

Figure 5 Calibration curve for 11.21 $\mathrm{M}$ benzene and $8.25 \mathrm{M}$ o-xylene solutions.

Table 3 Molecular sieving ability test data for AC and CMS.

\begin{tabular}{llccccc}
\hline Sample & Molecule & Initial absorbance & $\begin{array}{c}\text { Initial concentration } \\
/ \text { mol L }^{-1}\end{array}$ & $\begin{array}{c}\text { Absorbance at } \\
\text { equilibrium }\end{array}$ & $\begin{array}{c}\text { Equilibrium concentration } \\
/ \text { mol L }^{-1}\end{array}$ & Percentage uptake \\
\hline AC & Benzene & 0.7715 & 0.44 & 0.1679 & 0.08 & 98.41 \\
AC & O-Xylene & 0.7757 & 0.44 & 0.2447 & 0.13 & 70.45 \\
CMS 700/60 & Benzene & 0.7715 & 0.44 & 0.3084 & 0.17 & 61.36 \\
CMS 700/60 & O-Xylene & 0.7757 & 0.44 & 0.6360 & 0.36 & 18.18 \\
\hline
\end{tabular}

\subsection{Molecular Sieving Ability Test for AC and CMS 700/60}

A molecular sieving ability test was carried out on the AC and the CMS sample produced at $700{ }^{\circ} \mathrm{C}$ and $60 \mathrm{~min}$ deposition time (CMS 700/60), the results of which are presented in Table 3, while Fig. 5 presents the calibration curves for $11.21 \mathrm{M}$ benzene and $8.25 \mathrm{M}$ o-xylene solutions.

When the AC is brought into contact with a $0.44 \mathrm{~mol} \mathrm{~L}^{-1}$ binary solution of benzene and o-xylene, the percentage uptakes at equilibrium are both relatively high (98.41 and 70.45\%, respectively). This is because the AC performs rather poorly in its attempt to selectively adsorb either of the molecules over the other even though a marked drop in concentration can be observed for both molecules. This phenomena can be explained from the point of view that the benzene molecules having a kinetic diameter of $5.85 \AA^{20}$ being smaller than that of o-xylene (diameter $6.8 \AA$ ), ${ }^{21}$ was able to penetrate into the pore structure of the AC (HK pore size $9.44 \AA$ ) with a lot more ease than the larger o-xylene molecule. On the other hand, the CMS 700/60 (HK pore size $6.31 \AA$ ) performed better in terms of the selective discrimination of the benzene molecules over o-xylene. This is because the larger o-xylene molecules are unable to penetrate in to the CMS pore structure as readily as benzene. At this point it would be pertinent to note that CMS 700/60 does not possess a strictly uniform pore size distribution, meaning its pore structure consists of mesopores and pores with each sub-group having different pore size ranges. This is suggested in the work of Adinata et al. ${ }^{15}$ who established the heterogeneous pore size distributions of chemically activated coals and CMS even in cases where a significant portion of the distribution falls within the microporous range. This could be the reason why there was a noticeable uptake of o-xylene molecules at equilibrium despite it having a larger kinetic diameter than the pore size of the CMS 700/60 adsorbent.

\section{Conclusion}

A preliminary proximate analysis of the sample returned proximate and elemental analysis values confirming the bituminous nature of the Lafia-Obi Nasarawa coal used in this study. An AC was produced via chemical activation from the coal. The AC had a BET surface area of $596.2 \mathrm{~m}^{2} \mathrm{~g}^{-1}$, a DR pore volume of $0.173 \mathrm{~m}^{2} \mathrm{~g}^{-1}$ and a HK pore size of $9.432 \AA$. These results classify the $\mathrm{AC}$ as a microporous in nature capable of serving as a precursor for the production of CMS. Spent engine oil after undergoing pre-treatment was successfully used as a carbon depositor to achieve pore size modification of the AC to produce CMS under varying temperature and time regimes. The CMS produced designated CMS 700/60 in the text was found to have a BET surface area of $582.4 \mathrm{~m}^{2} \mathrm{~g}^{-1}$, DR pore volume of $0.172 \mathrm{~m}^{2} \mathrm{~g}^{-1}$ and HK pore size diameter of $6.317 \AA$. A molecular sieving ability test was carried out on AC and CMS 700/60 to test their ability to selectively adsorb benzene over o-xylene in a binary solution mixture. CMS 700/60 was successful to an extent in this regard and owing to its HK pore size (6.31 $\AA$ ) was determined to be a Type 2 carbon molecular sieve.

\section{${ }^{\S}$ ORCID iD}

E. Bala: iD orcid.org/0000-0002-3260-9430

\section{References}

1 A.A. Lizzao and R.M. Abadi, Production of carbon molecular sieves from Illinois coal, Fuel Pro. Tech., 1993, 34, 97-122.

2 R.L. Mieville and K.K. Robinson, Carbon Molecular Sieves and other Porous Carbons: Synthesis and Applications, 2015. http://www.megacarbon.com/techlit/carmolsiv.pdf

3 M. Mohammadi, A.R. Mohamed and G.D. Najafpour, Production of carbon molecular sieves from palm shell through carbon deposition from methane, Chem. Indus. Chem. Eng. Quat., 2011, 17(4), 525-5334. DOI: 10.2298/CICEQ110506038M

4 J. Alcañiz-Monge, J.P. Marco-Lozar and D. Lozano-Castelló, Mono- 
lithic carbon molecular sieves from activated bituminous coal impregnated with a slurry of coal tar pitch, Fuel Pro. Tech., 2012, 95, 67-72. DOI: 10.1016/j.fuproc.2011.11.016

5 D. Lozano-Castello, D. Cazorla-Amorós, A. Linares-Solano, W. Zhu, F. Kapteijn and J.A. Moulijn, Adsorption properties of carbon molecular sieves prepared by a co-carbonization process, Carbon, 2003, 43(8), 1643-1651.

DOI: 10.1016/j.carbon.2005.01.042

6. E. David, A. Talaieb, V. Stanciu and A.C. Nicolae, Synthesis of carbon molecular sieves by benzene pyrolysis over microporous carbon materials, Jour. Mat. Pro. Tech., 2004, 157-158, 290-296.

DOI: 10.1016/j.jmatprotec.2004.09.046

7 B.Y. Jibril, R.S. Al-Maamari, N. Al-Mandhary, G. Hegde and O. Houache, Effects of feedstock pre-drying on carbonization of $\mathrm{KOH}$-mixed bituminous coal in preparation of activated carbon, Jour. Anal. Appl. Pyrol., 2007, 80, 277-282.

DOI: 10.1016/j.jaap.2007.03.003

8 World Energy Council, World Energy Resources, 2016, 62. https://www.worldenergy.org/wp-content/uploads/2017/03/WERes ources_Coal_2016.pdf

9 Nigeria: Summary of the Coal Industry, 2014. https://www.globalmethane.org/documents/toolsres_coal_overview_ch24.pdf

10 D. Cuhadaroglu and A.U. Oznur, Production and characterization of activated carbon from a bituminous coal by chemical activation, Afr. J. Biotech., 2008, 7 (20), 3703-3710.

$11 \mathrm{H}$. Teng, T. Yeh and Hsu, Preparation of activated carbon from bituminous coal with phosphoric acid activation, Carbon, 1998, 36 (9), 1387-1395. http://dx.doi.org/10.1016/S0008-6223(98)00127-4

12 J.G. Speight, Handbook of Coal Analysis, 2005, Hoboken, John Wiley \& Sons Inc., New Jersey.

13 S.S. Lam, A.D. Russell and H.A. Chase, Pyrolysis using microwave heating: a sustainable process for recycling used car engine oil, Indus. Eng. Chem. Res., 2010, 49, 10845-10851. DOI: $10.1021 /$ ie100458f
14 S.S. Lam, A.D. Russell, C.L. Lee, S.K. Lam and H.A. Chase, Production of hydrogen and light hydrocarbons as a potential fuel from microwave-heated pyrolysis of waste automotive engine oil, Inter. J. Hydro. Energy, 2012, 37, 5011-5021. DOI: 10.1016/j.ijhydene.2011.12.016

15 D. Adinata, W.M. Daud and M.K. Aroua, Production of carbon molecular sieves from palm shell based activated carbon by pore sizes modification with benzene for methane selective separation, Fuel Proc. Tech., 2007, 88, 599-605. DOI: 10.1016/j.fuproc.2007.01.009

16 Y. Changtao, L. Yang, M. Yue, L. Shuyuan, H. Jilai and Q. Dengeke, Influence of retorting conditions on the pyrolysis of yaojie oil shale, Oil Shale, 2014, 31(1), 66-78. DOI: 10.3176/oil.2014.1.07

17 C. Briens, M. Latifi, L. Ferrante, I. Berruti and D. Radlein, Effects of Temperature and Residence Time on the Thermal Cracking of Bio-oil for Syngas Production, 2009. http://dc.engconfintl.org/bioenergy_ii/33

18 A.R. Mohamed, M. Mohammadi and G.N. Darzi, Preparation of carbon molecular sieve from lignocellulosic biomass: a review, Renew. Sust. Energy Rev., 2010, 14, 1591-1599. DOI: $10.1016 /$ j.rser.2010.01.024

19 X. Jiang, X. Wang, X. Han and J. Tong, Effect of residence time on products yield and characteristics of shale oil and gases produced by low-temperature retorting of dachengzi oil shale, Oil Shale, 2013, 30 (4), 501-516. DOI: 10.3176/oil.2013.4.04

$20 \mathrm{~J}$. Li and O. Talu, Effect of structural heterogeneity on multicomponent adsorption: benzene and p-xylene mixture on silicate, Funda. Adsor., 1993, 80, 373-380.

21 K.A.O. Santos, A.A. Dantas Neto, M.C.P.A. Moura and T.N. Castro Dantes, Separation of xylene isomers through adsorption on microporous materials: a review, Braz. J. Petrol. Gas., 2011, 5(1), 255-168.

DOI: $10.5419 /$ bjpg2011-0024 